\title{
An approximation algorithm for the Wireless Gathering Problem
}

\author{
Vincenzo Bonifaci $^{1,2 \star}$, Peter Korteweg ${ }^{1 \star \star}$, Alberto Marchetti-Spaccamela ${ }^{2}$, and \\ Leen Stougie ${ }^{1,3}$ \\ 1 Eindhoven University of Technology \\ Department of Mathematics and Computer Science \\ Den Dolech 2, 5600 MB Eindhoven, The Netherlands \\ v.bonifaci@tue.nl, p.korteweg@tue.nl, l.stougie@tue.nl \\ 2 University of Rome "La Sapienza" \\ Department of Computer and Systems Science \\ Via Salaria 113, 00198 Rome, Italy \\ bonifaci@dis.uniroma1.it, alberto@dis.uniroma1.it \\ 3 CWI, Kruislaan 413, 1098 SJ Amsterdam, The Netherlands \\ stougie@cwi.nl
}

\begin{abstract}
The Wireless Gathering Problem is to find a schedule for data gathering in a wireless static network. The problem is to gather a set of messages from the nodes in the network at which they originate to a central node, representing a powerful base station. The objective is to minimize the time to gather all messages. The sending pattern or schedule should avoid interference of radio signals, which distinguishes the problem from wired networks.

We study the Wireless Gathering Problem from a combinatorial optimization point of view in a centralized setting. This problem is known to be NP-hard when messages have no release time. We consider the more general case in which messages may be released over time. For this problem we present a polynomial-time on-line algorithm which gives a 4-approximation. We also show that within the class of shortest path following algorithms no algorithm can have approximation ratio better than 4. We also formulate some challenging open problems concerning complexity and approximability for variations of the problem.
\end{abstract}

\section{Introduction}

The last decade has seen a broad research focus on wireless networks $[9,10]$. Mobile phones, Bluetooth data communication and ad hoc laptop networks are testimony of the wide range of applications for wireless networks. Current interest on wireless sensor networks further emphasizes the importance of wireless networks in the future [8]. One of the main issues concerning wireless networks

\footnotetext{
* Work partly supported by Dutch Ministry of Education, Culture and Science through a Huygens scholarship.

** Work partly supported by EU COST-action 293.
} 
is data communication. Much research is focused on finding efficient communication protocols, i.e. protocols which minimize energy consumption or maximize throughput of the network.

In wireless networks stations communicate with each other through radio signals. A radio signal has a transmission radius, the distance over which the signal is strong enough to send data and an interference radius, the distance over which the radio signal is strong enough to interfere with other radio signals.

Interference, also called collision, and fading of radio signals are severe problems in wireless networks and the main features that distinguish communication in wireless networks from communication in wired networks. Interference is the effect of radio signal loss due to the fact that multiple stations located within interference radius of each other try to communicate simultaneously. Fading is the effect of radio signal loss due to physical circumstances. Interference and fading cause data loss which results in lower throughput or equivalently higher completion times [12]. Furthermore, it causes higher energy consumption as data has to be resent.

Communication protocols can be divided into five different layers [11]: the application layer, the transport layer, the network layer, the data link layer and the physical layer. The transport layer is used to provide a data delivery protocol between the stations. The network layer is concerned with routing of data supplied by the transport layer. In order to route data, stations need to have information on the structure of the wireless network. See [10] for an overview of routing protocols which provide such information. The data link layer is concerned with finding medium access (MAC) schemes; such a scheme determines which nodes send data at a certain time.

MAC schemes can be characterized as either fixed assignment schemes or contention schemes [9]. A fixed assignment scheme provides an allocation of medium access resources; these resources are time and frequency. This leads to Time Division Multiple Access (TDMA) schemes where each station is allocated a time slot to send data. Similarly there are Frequency Division Multiple Access (FDMA) schemes where each station is allocated a frequency range to send data and Code Division Multiple Access (CDMA) schemes which is a combination of both time and frequency allocation. As resource allocation requires coordination fixed assignment schemes are common in a centralized setting.

Contention schemes are schemes where stations compete for medium access. In a contention scheme stations access the medium independently of other stations. The most common contention schemes are Carrier Sense Multiple Access (CSMA) schemes. Because stations compete to access the medium there is the possibility of collision. There are several techniques for collision detection (CD) or collision avoidance (CA). The 802.11 protocol [7], a widely used standard for Wireless Local Area Networks describes both a fixed assignment scheme and a contention scheme: the point coordination function (PCF) in a centralized setting and the distributed coordination function (DCF).

In this paper we consider the Wireless Gathering Problem (WGP). The WGP is to find a schedule for data gathering at a base station of a wireless static net- 
work which minimizes gathering time of messages. Gathering data at some base station is a well known process in wireless networks and is sometimes referred to as many-to-one communication. Typically, base stations are powerful stations which are able to process the data and act as gateways to other networks [6].

The Wireless Gathering Problem is the following: given is a static wireless network which consists of several stations (nodes) and one base station (the sink). Some stations contain a set of packets (messages) which have to be gathered at the base station. Wireless communication enables stations to communicate data packets to each other. We assume that time is discrete and that stations have a common clock, hence time can be divided into rounds. In our model a node may either send or receive a single packet during a round. Typically, not all nodes in the network can communicate with each other, hence packets have to be sent through several nodes before they can be gathered at the sink; this is called multihop routing. The problem consists of constructing a schedule without interference which determines for each packet both route and times at which it is sent. The objective of the problem is to find a schedule which minimizes a function of the completion times of the packets, i.e., the times at which the packets arrive at the sink. We consider minimization of maximum completion time.

The WGP combines the problem of data routing at the network layer and scheduling at the data link layer. We assume that the stations are provided with the necessary information on the graph structure, such as path distances. Thus we study the problem in a centralized setting. We also assume perfect radio signals, i.e. no signal loss due to fading. We consider both a version where messages have no release time, i.e. are released at time zero, and a version in which messages are released over time.

Much research has been devoted to the design of communication protocols, but most models do not consider interference. See [5] for a general overview of communication protocols. In the centralized setting without release times, the many-to-one gathering problem is closely related to the one-to-all personalized broadcast, where the base station wants to communicate a unique message to each station in the network. In [1] and [2] the authors study the one-to-all (nonpersonalized) broadcast on arbitrary graphs in a model similar to ours, for the case where both transmission radius and interference radius are unit distance. In the second paper the authors consider the WGP as a subproblem. For this problem they give a distributed randomized algorithm with expected completion time of $O((n+\delta) \log d)$, where $n$ is the number of messages, $\delta$ the diameter of the graph and $d$ the maximum degree. In [4] the authors give an optimal centralized greedy algorithm for the gathering problem without interference on a tree.

The Wireless Gathering Problem with arbitrary integral transmission and interference radius was formulated as a combinatorial optimization problem by Bermond et al. [3]. In this paper the authors prove that WGP is NP-hard even without release times (or equivalently, when all messages are released at time zero). For this case they present an approximation algorithm and sketch a proof that it gives an asymptotic 4-approximation and an asymptotic 3-approximation if transmission radius is equal to interference radius. The authors do not consider 
the case in which messages are released over time. In Section 2 we formulate the problem precisely.

In Section 3 we present an on-line polynomial-time greedy algorithm which for arbitrary release times gives a 4-approximation in general and a 3approximation when transmission radius and interference radius are equal. Our results improve over those of Bermond et al. [3] only slightly in case all messages are released at time zero. However, our algorithm is simple, and the approximation ratio indeed holds for arbitrary release times. Both our algorithm and that of Bermond et al. send all messages along the shortest path to the sink. We prove that within the class of shortest path following algorithms no algorithm can have approximation ratio better than 4 . Thus, within this class our algorithm is best possible. Furthermore, we prove that our algorithm is optimal on a chain with unit transmission radius and without release times when the sink is at one end of the chain. The complexity of all other variations of the problem on a chain and on a tree is to the best of our knowledge still open. These and some other challenging research opportunities on the Wireless Gathering Problem conclude the paper in Section 4.

\section{Mathematical formulation}

We formulate the WGP as a graph optimization problem. Given is a graph $G=(V, E)$ with $|V|=n$, sink $s \in V$, and a set of messages (data packets) $M=\{1,2, \ldots, m\}$. If $(u, v) \in E$ then $u$ and $v$ can communicate directly with each other. We assume that each edge has unit length. For each pair of nodes $u, v \in V$ we define the distance between $u$ and $v$, denoted by $d(u, v)$, as the length of a shortest path from $u$ to $v$ in $G$. We introduce $d_{I}$ as the interference radius and $d_{T}$ as the transmission radius. Each message $j \in M$ has an origin $v_{j} \in V$.

We assume that time is discrete, say $\{0,1, \ldots\}$; we call a time unit a round. The rounds are numbered $0,1, \ldots$. During each round a node may either be sending a message, be receiving a message or be inactive. If $d(u, v) \leq d_{T}$ then $u$ can send some message $j$ to $v$ during a round. If node $u$ sends a message $j$ to $v$ in some round, then the pair $(u, v)$ is called a call of message $j$ during that round. Two calls $(u, v)$ and $\left(u^{\prime}, v^{\prime}\right)$ interfere if $d\left(u^{\prime}, v\right) \leq d_{I}$ or $d\left(u, v^{\prime}\right) \leq d_{I}$; otherwise the calls are compatible.

The solution of the WGP is a schedule of compatible calls such that all messages are sent to the sink. Given a schedule, let $v_{j}^{t}$ be the node of message $j$ at time $t . C_{j}=\min \left\{t: v_{j}^{t}=s\right\}$ is called the completion time of message $j$. We consider the minimization of $\max _{j} C_{j}$ (makespan). We assume that messages cannot be aggregated. Every message $j$ has a release time $r_{j} \in \mathbb{Z}_{+}$at which it enters the network. We consider the case in which all messages are released at the same time, i.e. $r_{j}=0$ for all $j$, as a special case. In the off-line version all message information is known at time 0 , in the on-line version information about a message becomes known only at its release time. The off-line WGP 
is equivalent to a one-to-many personalized broadcast problem: a time reverse gathering schedule provides a one-to-many personalized broadcast schedule.

\section{A greedy algorithm}

We present a greedy algorithm which assigns messages to calls according to some priority ordering. Each message is assigned to a call of maximum distance without causing interference. Let $\phi(j)=\left\lceil\frac{d\left(v_{j}, s\right)}{d_{T}}\right\rceil$, the minimum number of calls required for $j$.

Priority Greedy (PG). Given messages $j$ and $k$, we say that $j$ has higher priority than $k$ if $r_{j}+\phi(j)$ is smaller than $r_{k}+\phi(k)$, ties broken arbitrarily. In every round, consider the available messages in order of decreasing priority, and send each next message as far as possible along a (possibly prefixed) shortest path from its current node to $s$, without creating interference with any higherpriority message.

Notice that PG is an on-line, polynomial-time algorithm. To analyze the worstcase approximation ratio of $P G$ we first derive upper bounds on the completion time of each message in a PG solution.

Given a message $j$, we say that $j$ is blocked in round $t$ if, in round $t, j$ cannot be sent over distance $d_{T}$ towards $s$ (or it cannot be sent to $s$ if $d\left(v_{j}^{t}, s\right) \leq d_{T}$ ) because of interference with some higher-priority message in PG.

We define the following blocking relation on a PG schedule: $k \prec j$ if in the last round in which $j$ is blocked, $k$ is the message closest to $j$ that is sent in that round and a priority higher than $j$ (ties broken arbitrarily). The blocking relation induces a directed graph $F=(M, A)$ on the message set $M$ with an arc $(k, j)$ for each $k, j \in M$ such that $k \prec j$. Observe that for any PG schedule $F$ is a directed forest and the root of each tree of $F$ is a message which is never blocked. For each $j$ let $T(j) \subseteq F$ be the tree of $F$ containing $j, b(j) \in M$ be the root of $T(j)$, and $P(j)$ the path in $F$ from $b(j)$ to $j$. Let $h(j)$ be the length of $P(j)$. Finally, define $\gamma=1+\left\lceil\frac{d_{I}+1}{d_{T}}\right\rceil$ and let $C_{j}$ denote the completion time of message $j$ in a PG schedule.

Lemma 1. For each message $j \in M$,

$$
C_{j} \leq r_{b(j)}+\phi(b(j))+\sum_{i \in P(j), i \neq b(j)} \min \{\phi(i), \gamma\} .
$$

Proof. The proof is by induction on $h(j)$. Any message $j$ with $h(j)=0$ is never blocked, hence $b(j)=j$, and the lemma is obviously true.

Otherwise, let $t$ be the last round in which $j$ is blocked by some message $k$, $k \prec j$. By definition of the blocking relation we have $d\left(v_{j}^{t}, v_{k}^{t}\right) \leq d_{T}+d_{I}$ and if $d\left(v_{j}^{t}, v_{k}^{t}\right)>d_{I}+1$ then $j$, although blocked, is sent to $v_{j}^{t+1}$ with $d\left(v_{j}^{t+1}, v_{k}^{t}\right)=$ $d_{I}+1$. Also, $d\left(v_{k}^{t}, s\right) \leq\left(C_{k}-t\right) d_{T}$, otherwise $k$ would not reach $s$ by time $C_{k}$. 
From time $t+1$ on, $j$ is forwarded to $s$ over distance $d_{T}$ each round, reaching $s$ at

$$
\begin{aligned}
C_{j} & \leq t+1+\left\lceil\frac{d\left(v_{k}^{t}, s\right)+d\left(v_{j}^{t+1}, v_{k}^{t}\right)}{d_{T}}\right\rceil \\
& \leq t+1+C_{k}-t+\left\lceil\frac{d_{I}+1}{d_{T}}\right\rceil \\
& =C_{k}+1+\left\lceil\frac{d_{I}+1}{d_{T}}\right\rceil=C_{k}+\gamma .
\end{aligned}
$$

Also, $C_{j} \leq C_{k}+\phi(j)$, since after $k$ reaches $s, j$ will need no more than $\phi(j)$ rounds to reach $s$. Thus $C_{j} \leq C_{k}+\min \{\phi(j), \gamma\}$ and the lemma follows by applying the induction hypothesis to $C_{k}$.

Now we derive lower bounds on the optimal cost. We define the critical radius $R^{*}$ as the greatest integer $R$ such that no two nodes at distance at most $R$ from $s$ can receive a message in the same round. Notice that $R^{*} \geq\left\lfloor\frac{d_{I}-d_{T}}{2}\right\rfloor$. The critical region is the set of nodes at distance at most $R^{*}$ from $s$. Define $\gamma^{*}=\left\lceil\frac{R^{*}+1}{d_{T}}\right\rceil$. Let $C_{j}^{*}$ denote the completion time of message $j$ in an optimal solution.

Lemma 2. Let $S \subseteq M$ be a nonempty set of messages. Then there is $k \in S$ such that

$$
\max _{j \in S} C_{j}^{*} \geq r_{k}+\phi(k)+\sum_{j \in S, j \neq k} \min \left\{\phi(j), \gamma^{*}\right\} .
$$

Proof. Define $p_{j}=\min \left\{\phi(j), \gamma^{*}\right\}$ and $r_{j}^{\prime}=r_{j}+\phi(j)-p_{j}$. Since in every round at most one message can move inside the critical region, any feasible solution to the Wireless Gathering Problem gives a feasible solution to a preemptive single machine scheduling problem in which the release time of job $j$ (corresponding to message $j$ ) is $r_{j}^{\prime}$ and its processing time is $p_{j}$. By ignoring interference outside the critical region we can only decrease the optimum cost, thus a lower bound on the scheduling cost is also a lower bound on the gathering cost.

Now let $k$ be the first message in $S$ entering or being released in the critical region in the optimal schedule. In the scheduling relaxation, the makespan is at least the time at which the first job starts processing plus the sum of the processing times:

$$
\begin{aligned}
\max _{j \in S} C_{j}^{*} & \geq r_{k}^{\prime}+\sum_{j \in S} p_{j} \\
& =r_{k}+\phi(k)+\sum_{j \in S, j \neq k} p_{j} .
\end{aligned}
$$

Theorem 1. PRIORITY GREedy gives a $\max \left\{2, \gamma / \gamma^{*}\right\}$-approximation to the Wireless Gathering Problem with release times. When $r_{j}=0$ for all $j \in M$, PRIORITY GREeDY gives a $\gamma / \gamma^{*}$-approximation. 
Proof. Let $j$ be the message having maximum $C_{j}$, and consider $T(j)$, the tree containing $j$ in the forest induced by the blocking relation. We can apply Lemma 2 with $S=T(j)$ to obtain

$$
\max _{i \in T(j)} C_{i}^{*} \geq r_{k}+\phi(k)+\sum_{i \in T(j), i \neq k} \min \left\{\phi(i), \gamma^{*}\right\}
$$

where $k$ is some message in $T(j)$. On the other hand, by using Lemma 1 ,

$$
\begin{aligned}
C_{j} & \leq r_{b(j)}+\phi(b(j))+\sum_{i \in P(j), i \neq b(j)} \min \{\phi(i), \gamma\} \\
& \leq r_{b(j)}+\phi(b(j))+\min \{\phi(k), \gamma\}+\sum_{i \in P(j), i \neq b(j), i \neq k} \min \{\phi(i), \gamma\} \\
& \leq 2\left(r_{k}+\phi(k)\right)+\frac{\gamma}{\gamma^{*}} \sum_{i \in T(j), i \neq k} \min \left\{\phi(i), \gamma^{*}\right\}
\end{aligned}
$$

where we used the fact that $b(j)$, being the root of $T(j)$, minimizes $r_{i}+\phi(i)$ in $T(j)$. Now let $\alpha \geq 0$ be such that $\sum_{i \in T(j), i \neq k} \min \left\{\phi(i), \gamma^{*}\right\}=\alpha\left(r_{k}+\phi(k)\right)$. Then

$$
\frac{C_{j}}{\max _{i \in T(j)} C_{i}^{*}} \leq \frac{2+\frac{\gamma}{\gamma^{*}} \alpha}{1+\alpha} \leq \max \left\{2, \gamma / \gamma^{*}\right\}
$$

For the case in which $r_{j}=0$ for all $j \in M$, we proceed similarly, but also notice that since now $b(j) \in \operatorname{argmin}_{i \in T(j)}\{\phi(i)\}$, we have $\phi(b(j)) \leq \phi(k)$ and the claim follows by directly comparing (1) with (2).

Corollary 1. Priority Greedy is 3-approximate if $d_{I}=d_{T}$ and 4approximate in general for WGP with release times.

Proof. We distinguish several cases:

Case 1 If $d_{I} \leq 2 d_{T}-1$ then $\gamma=3$, which in particular proves the 3 -approximation in case $d_{I}=d_{T}$.

Case 2: $d_{I} \leq 3 d_{T}-1$. Then $\gamma \leq 4$.

Case 3: $l d_{T} \leq d_{I} \leq(l+2) d_{T}-1$ for any odd integer $l \geq 3$. Then $\gamma \leq l+3$ and as $r^{*} \geq\left\lfloor\frac{d_{I}-d_{T}}{2}\right\rfloor$ it follows that $\gamma^{*} \geq(l+1) / 2$, hence $\gamma / \gamma^{*} \leq 2(l+3) /(l+1) \leq 3$.

The analysis shows that the ratio $\gamma / \gamma^{*}=4$ only if $d_{I} / d_{T} \in[2,3)$ and the ratio approaches 2 if $d_{I} / d_{T}$ tends to infinity.

Corollary 2. When $r_{j}=0$ for all $j \in M$, PRIORITY GREEDY is optimal if $G$ is a chain with $s$ as an extreme and $d_{T}=1$.

Proof. If $G$ is a chain and $s$ an extreme, then the critical radius is $d_{I}+1$. Thus, for $d_{T}=1$ we have $\gamma^{*}=d_{I}+2$. The claim follows since $\gamma=d_{I}+2$ for $d_{T}=1$ and without release times the approximation ratio is $\gamma / \gamma^{*}$. 
PG sends messages over shortest paths. We show that no algorithm which sends each message $j$ over a shortest path from $v_{j}$ to $s$ can be better than 3approximate if $d_{I}=d_{T}$ and 4-approximate if $d_{I}>d_{T}$. This means that to find algorithms with lower approximation ratios than PG messages need to be diverged from their shortest path to the sink if this path becomes congested.

First, consider the example of Figure 1 with $d_{I}=d_{T}=1$. Nodes $u_{1}, u_{2}, u_{3}$ have $m / 3$ messages each. Any shortest paths following algorithm sends all messages via $u$, yielding $\max _{j} C_{j}=3 \mathrm{~m}$. On the other hand there is a solution with no message passing $u$ that implies $\max _{j} C_{j}^{*} \leq 3+m$. The example can easily be extended for arbitrary $d_{I}=d_{T}$ such that GREEDY is a 3-approximation.

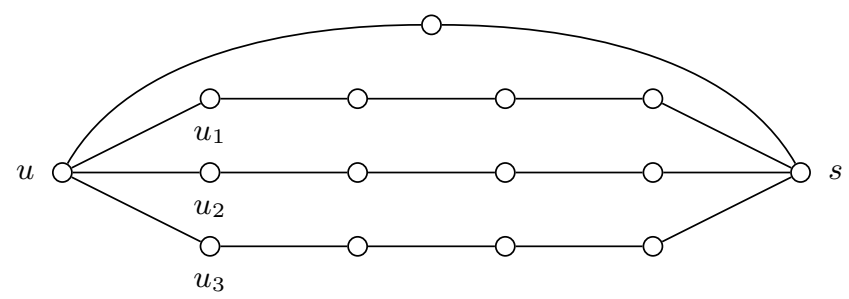

Fig. 1. Any shortest paths following algorithm is no better than a 3-approximation for $d_{I}=d_{T}=1$.

In case $d_{I}>d_{T}$ consider Figure 2 . The nodes $u_{1}, \ldots, u_{m}$ each have 1 message. Let $d_{I}=2$ and $d_{T}=1$. Any shortest paths following algorithm sends all messages via $u$, yielding $\max _{j} C_{j}=4 m$. There is a solution with no message passing $u$ that implies $\max _{j} C_{j}^{*} \leq 4+m$. The example can easily be extended for arbitrary $d_{I}=2 d_{T}$ such that GREEDY is a 4 -approximation.

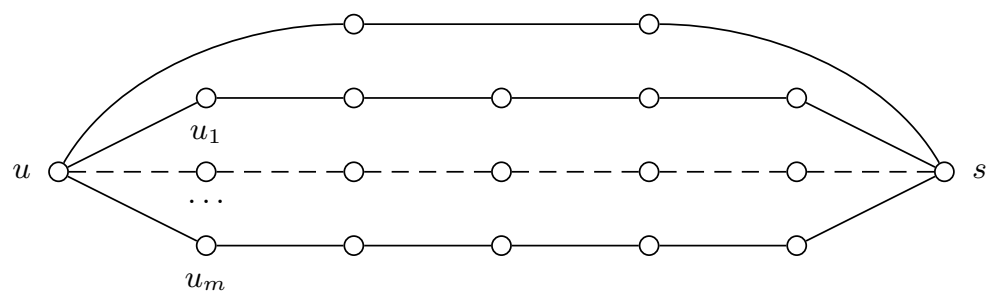

Fig. 2. Any shortest paths following algorithm is no better than a 4-approximation for $d_{I}=2, d_{T}=1$.

In these examples the optimal schedule sends each message over a path of length exceeding the length of its shortest path by at most 1 . This may suggest to consider algorithms which send messages over paths whose length does not exceed their shortest path length by some constant $k$. However, as can easily 
be verified, for each constant $k$ we could change the length of the paths in the examples above, such that the optimal schedule sends each message over a path whose length exceeds the shortest path length by $k+1$.

Improvement on the approximation ratio should come from algorithms that avoid congested paths. One such an idea is to use not only the shortest path but the $k$ shortest paths, whichever of them is least congested. By adapting Figure 1, the example in Figure 3 is obtained, showing for $k=2$ that even if we choose the 2 shortest internally vertex disjoint paths, the lower bound on the approximation ratio for $d_{I}=d_{T}$ remains unchanged. The example can be extended to any fixed $k$. Similarly the example of Figure 2 can be adapted to show that in case $d_{I}>d_{T}$ choosing any of $k$ shortest paths, for fixed $k$, leaves the lower bound of 4 on the ratio unchanged.

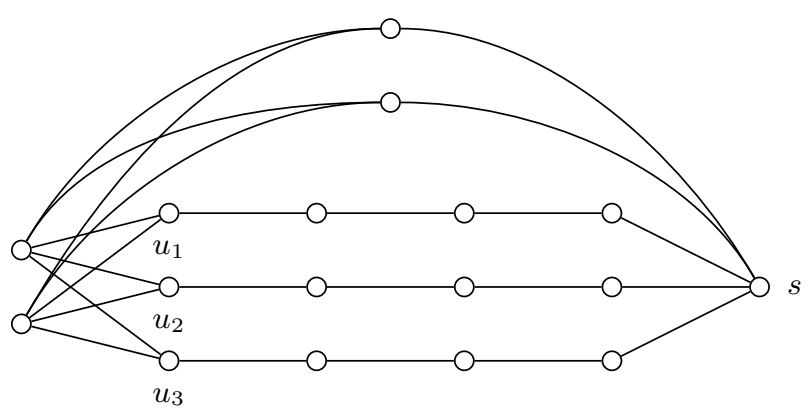

Fig. 3. Any shortest paths following algorithm using 2 internally vertex disjoint shortest paths is no better than a 3-approximation for $d_{I}=d_{T}=1$.

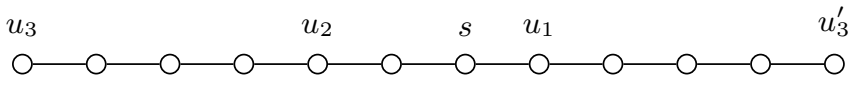

Fig. 4. $P G$ is not optimal on a chain if the sink is not at an extreme.

PG can be non-optimal on a chain if the sink is not at an extreme node of the chain. Consider the instance given by the graph in Figure 4 . Let $d_{T}=1$ and $d_{I}=2$ and assume messages are released in $u_{1}, u_{2}$ and $u_{3}$ at time zero. PG would first send the message in $u_{1}$ to the sink, and then send the message in $u_{2}$ to the sink, resulting in a makespan of 7 , while in an optimal solution the messages in $u_{2}$ and $u_{3}$ are forwarded until the message of $u_{2}$ reach the sink at time 2, then the messages of $u_{3}$ and $u_{1}$ are forwarded simultaneously, yielding a cost of 6 . However, if the third message would have been released at node $u_{3}^{\prime}$ instead of at node $u_{3}$ then PG would have been optimal. This shows that any optimal algorithm for the problem on a chain should take into account the position of 
the other messages when deciding which of the two messages nearest to the sink to send first. The complexity of the problem on a chain remains open.

\section{Challenging Future Research Problems}

In this paper we designed and analyzed algorithms for a basic centralized gathering problem (WGP) on a wireless static network with unit edge lengths. Specifically, we proved that a greedy algorithm has approximation ratio 4 for this WGP when minimizing maximum completion time. We also showed that our greedy algorithm yields the best possible approximation ratio within the class of algorithms in which each message is sent over a shortest path to the sink. It is a beautiful challenge to design algorithms that avoid congested paths, which have approximation ratios strictly less than 4 (or 3 if $d_{I}=d_{T}$ ). Our examples at the end of the previous section show that this is not so trivial. For instance selecting among $k$ shortest paths (even disjoint) will not give lower ratios. On the other hand, from the proof of Corollary 1, one could concentrate on the subclass of problems with $d_{I} / d_{T} \in[2,3)$ to improve on the ratio of 4 , since in all other cases greedy has ratio at most 3 .

All our results apply to general graphs; we have not considered specific graphs in depth. Specifically, the complexity of WGP on chains or trees is open, apart from the restricted case in Corollary 2. Our suspicion is that these problems are easy as well, but the greedy algorithm is not optimal on a chain for $d_{T}=1$ and $d_{I}=2$ as we have shown at the end of the previous section. The example can easily be extended to any combination of $d_{I}$ and $d_{T}$, except $d_{I}=d_{T}$, for which greedy might be optimal. Also, our attempts to formulate the problem on a chain as a dynamic programming problem have failed so far.

It is interesting to study variations of the problem. An evident generalization would be to use an arbitrary distance function, instead of unit distances. We strongly believe that our algorithm is a constant approximation algorithm for arbitrary distance functions, though the approximation ratio may be worse. Although we did not prove it here, we have shown by similar arguments as used in this paper that for the problem without release times our algorithm also gives a 4-approximation when the objective function is the sum of completion times, and that this ratio is best possible among shortest path following algorithms. We will report on this result in a research report version of the paper.

A real challenge is to study a much more realistic version of the problem: the real problem is on-line with distributed algorithms at each of the nodes. In on-line WGP a message becomes known only at its release time and the sending schedules have to be adapted to newly released messages in an on-line fashion. In fact, our greedy algorithm is an on-line algorithm, though centralized, and thus gives a competitive ratio of 4 . However, there is a significant gap between this upper bound and a simple lower bound on the competitive ratio of any deterministic centralized algorithm: we have constructed rather simple examples which give lower bounds of $7 / 5$ for $d_{I}>d_{T}$ and $4 / 3$ for $d_{I}=d_{T}$. 
In distributed WGP coordination of communication is very limited. As interference can not always be detected a priori in this model, algorithms should be able to accommodate for retransmissions of lost data. Bar-Yehuda et al. [2] designed distributed randomized algorithms that do this for WGP with $d_{T}=d_{I}=1$ without release times (thus in an off-line setting). They derive bounds on the expected number of rounds required to gather all messages at the sink. It would be interesting to exploit ideas in this paper to design (randomized) distributed on-line algorithms and obtain satisfactory competitive ratios. It may very well be that constant competitive ratios are not achievable.

\section{References}

1. R. Bar-Yehuda, O. Goldreich, and A. Itai. On the time-complexity of broadcast in multi-hop radio networks: an exponential gap between determinism and randomization. Journal of Computer and System Sciences, 45(1):104-126, 1992.

2. R. Bar-Yehuda, A. Israeli, and A. Itai. Multiple communication in multihop radio networks. SIAM Journal on Computing, 22(4):875-887, 1993.

3. J. Bermond, J. Galtier, R. Klasing, N. Morales, and S. Perennes. Hardness and approximation of gathering in static radio networks, 2006. Manuscript.

4. S. N. Bhatt, G. Pucci, A. Ranade, and A. L. Rosenberg. Scattering and gathering messages in networks of processors. IEEE Trans. Comput., 42(8):938-949, 1993.

5. P. Fraigniaud and E. Lazard. Methods and problems of communication in usual networks. Discrete Appl. Math., 53:79-133, 1994.

6. M. Haenggi. Opportunities and challenges in wireless sensor networks. In Handbook of Sensor Networks: Compact Wireless and Wired Sensing Systems. CRC Press, Boca Raton, 2004.

7. IEEE Standard 802.11, Information technology-Telecommunications and information exchange between systems-Local and metropolitan area networks-Specific requirements-Part 11: Wireless LAN Medium Access Control (MAC) and Physical Layer (PHY) Specifications, 1999.

8. M. Ilyas and I. Mahgoub. Handbook of Sensor Networks: Compact Wireless and Wired Sensing Systems. CRC Press, Boca Raton, 2004.

9. K. Pahlavan and A. H. Levesque. Wireless information networks. WileyInterscience, New York, NY, USA, 1995.

10. C. E. Perkins. Ad hoc networking. Addison-Wesley Longman Publishing Co., Inc., Boston, MA, USA, 2001.

11. W. Su, E. Cayirci, and O. Akan. Overview of communication protocols for sensor networks. In Handbook of Sensor Networks: Compact Wireless and Wired Sensing Systems. CRC Press, Boca Raton, 2004.

12. D. Tse and P. Viswanath. Fundamentals of Wireless Communication. Cambridge University Press, Cambridge, 2005. 